\title{
Odd-elegant Labeling Algorithm of Generalized Ring Core Networks
}

\author{
Jianmin Xie ${ }^{1, a}$, Bing Yao ${ }^{2, b}$ and Wenmei Hong ${ }^{3, c}$ \\ ${ }^{1}$ College of Mathematics, Lanzhou City University, Lanzhou, China \\ ${ }^{2}$ College of Mathematics and Statistics, Northwest Normal University, Lanzhou, China \\ ${ }^{3}$ College of Early Childhood Teachers, Lanzhou City University, Lanzhou, China \\ a Email:xjm7601213@163.com, ${ }^{b}$ Email:yybb918@163.com, ${ }^{c}$ Email:hongwm2007@163.com
}

Keywords: generalized ring core network; odd- elegant feature; odd- elegant labeling; odd- elegant labeling algorithm; effectiveness of algorithm

\begin{abstract}
In the network design, the choice of the network topology plays a decisive role for the realization of the function of computer network efficiency. The labeling problem of the computer network topology directly affects the network design and communication costs, etc. Generalized ring network topology is a very important hybrid network topology structure, and generalized ring core network is its base. In this paper, based on the requirements of research of generalized ring network addressing, the author designs the GRN-OEL-algorithm when $n_{1}, n_{2}, \cdots, n_{m} \equiv 0(\bmod 4)$, proves odd-elegant of the generalized ring core network, works out the corresponding software, and tests the practical effectiveness of this algorithm with our experimental data.
\end{abstract}

\section{Introduction}

Computer network is the result of the close combination, mutual penetration, mutual promotion, and common development between computer technology and communication technology. In the network design, the choice of the network topology plays a decisive role for the realization of the function of computer network efficiency. The labeling problem of the computer network topology directly affects the network design and communication costs, etc. The labeling of network topology refers to a mapping of integer set to the node or edge of network topology, and it satisfies certain conditions. According to different conditions, the researchers defined several types of labeling of network topology and put forward many conjectures. In 1981, Chang put forward the concept of elegant labeling and guessed that [1]: all tree topology are elegant. In 2013, Zhou et al., defined the concept of odd-elegant labeling and put forward the hypothesis that [2]: all tree topology are odd-elegant. Bus topology, star topology and ring network topology are the three basic network topologies, and the labeling problems of them lays a necessary theoretical basis for the design of the computer network system. However, the singleness of their structure seriously affects the extension of network function. Therefore, the new topology structure has been organic combined with two or more a single topology structure, which, has become an important research topic for researchers of computer theory and application, especially for the web worker[3-6]. In 2007, Gao Zhenbin proved the odd-graceful of three union structure $\bigcup P_{n}, \bigcup S_{n}$ and $\bigcup C_{n},(n$ is a multiple of 4) [7]. In 2009, Barrientons proved the odd-graceful of topology structure of tree with its diameter of no more than five [8]. In 2013, Zhou et al. proved the odd-elegant of lobster -----a hybrid topology structure [2].

Generalized ring network is a very important hybrid network topology structure, which refers to a number of closed rings formed of nodes linked together point to point and end to end in network and each nodes of rings has a line nodes(we call line nodes leaves). The information both can be translated in a direction between nodes in the loop, and can be translated between ring nodes and leaves. After the leaves of generalized ring network are removed, we call the rests the generalized ring core network. Obviously, generalized ring core network is the basis of generalized ring network. In this paper, based on the requirements of research of generalized ring network addressing, the author designs the GRN-OEL algorithm when $n_{1}, n_{2}, \cdots, n_{m} \equiv 0(\bmod 4)$, proves odd-elegant of this 
network topology, works out the corresponding software, and tests the practical effectiveness of this algorithm with our experimental data.

\section{Preliminary knowledge}

We begin with simple, finite and undirected network topology $G=(V, E)$ with $V$ for nodes set and $E$ for edges set. For the network topology $G$, positive integer $p$ is called as the nodes number of $G$, and $q$ is called as the edges number of $G$. For the sake of simplicity, the shorthand symbol $[m, n]$ stands for an integer set $\{m, m+1, \cdots, n\}$, where $m$ and $n$ are integers with $0 \leq m<n$.

Definition 1 [2]. A function $f$ is called odd-elegant labeling of a graph $G$ if $f: V \rightarrow[0,2 q-1]$ is injective and the induced function $f^{*}: E(G) \rightarrow\{1,3, \cdots, 2 q-1\}$ defined as $f^{*}(e)=|f(u)+f(v)| \bmod 2 q$, $(e=u v)$ is bijection. The graph which admits odd-elegant labeling is called an odd-elegant graph.

Definition 2. Let $m$ is a integer not less than 2 , and let $n_{1}, n_{2}, \cdots, n_{m} \equiv 0(\bmod 4)$ are all positive integer. The nodes in each ring $C_{n_{i}}(i \in[1, m])$ will be ordered into $v_{i 1}, v_{i 2}, \cdots, v_{i n_{i}}$ in the same direction. And let the node $v_{i\left(n_{i}-1\right)}$ of $n_{i}-1$ of ring $C_{n_{i}}(i \in[1, m])$ is coincided with first node $v_{(i-1) 1}$ of ring $C_{n_{i-1}}$, then a network topology $G=\omega_{n_{1} n_{2} \cdots n_{m}}$ including $m$ rings is defined, which is called as generalized ring core network if $n_{1}, n_{2}, \cdots, n_{m} \equiv 0(\bmod 4)$ (figure 1$)$.

\section{Odd-elegant labeling algorithm of generalized ring core network}

Let $G=\omega_{n_{1} n_{2} \cdots n_{m}}$ be a generalized ring core network if $m, n_{1}, n_{2}, \cdots, n_{m} \in N^{*} \quad$ and $n_{1}, n_{2}, \cdots, n_{m} \equiv 0 \quad(\bmod 4) \quad$, then: $V=\left\{v_{11}\left(v_{2\left(n_{2}-1\right)}\right), v_{12}, \cdots, v_{1\left(n_{1}-1\right)}, v_{1 n_{1}}, v_{21}\left(v_{3\left(n_{3}-1\right)}\right), v_{22}, \cdots\right.$, $v_{2\left(n_{2}-2\right)}, \quad v_{2 n_{2}}, \cdots, v_{(m-1) 1}\left(v_{m\left(n_{m}-1\right)}\right), \quad v_{(m-1) 2}, \quad v_{(m-1) 3}$, $\left.\cdots, v_{(m-1)\left(n_{m-1}-2\right)}, v_{(m-1) n_{m-1}}, \quad v_{m 1}, v_{m 2}, \cdots, v_{m\left(n_{m}-2\right)}, v_{m n_{m}}\right\}$, and $p=\sum_{k=1}^{m} n_{k}-m+1, \quad q=\sum_{k=1}^{m} n_{k}$.

The node been overlap labeling is regarded as two different nodes $v_{(i-1) 1}$ and $v_{i\left(n_{i}-1\right)}(i \in[2, m])$. All nodes can be divided into three parts:

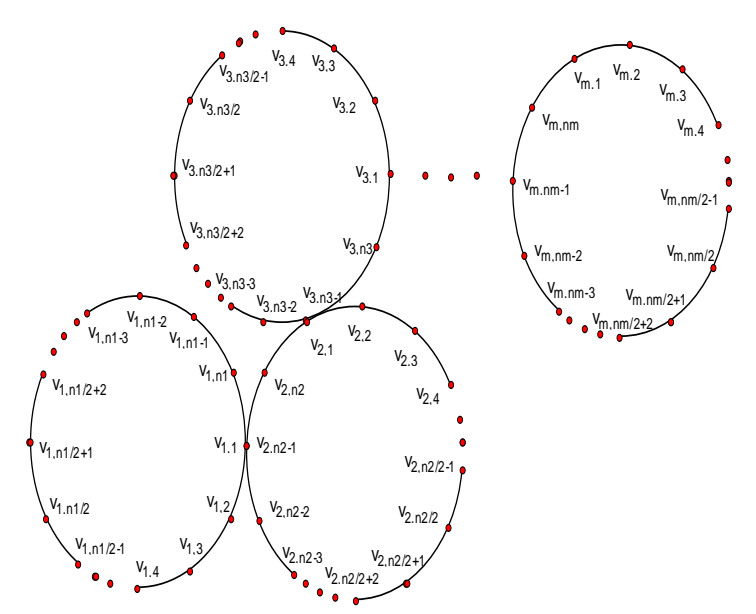

Fig. 1. Generalized ring core network with labeled vertices

$V_{1}=\left\{v_{i(2 j-1)} \mid i \in[1, m], j \in\left[1, \frac{n_{i}}{4}\right]\right\}, V_{2}=\left\{v_{i(2 j-1)} \mid i \in[1, m], j \in\left[\frac{n_{i}}{4}+1, \frac{n_{i}}{2}\right]\right\}, V_{3}=\left\{v_{i(2 j)} \mid i \in[1, m], j \in\left[1, \frac{n_{i}}{2}\right]\right\}$.

Edge set $E=\left\{v_{i j} v_{i(j+1)}, v_{i n_{i}} v_{i 1} \mid i \in[1, m], j \in\left[1, n_{i}-1\right]\right\}$ according to the location of ring can be divided into $m$ parts:

$$
\begin{gathered}
\quad E_{1}=\left\{v_{11} v_{12}, v_{12} v_{13}, \cdots, v_{1\left(n_{1}-1\right)} v_{1 n_{1}}, v_{11} v_{1 n_{1}}\right\} \quad, \quad E_{2}=\left\{v_{21} v_{22}, v_{22} v_{23}, \cdots, v_{2\left(n_{2}-1\right)} v_{2 n_{2}}, v_{2 n_{2}} v_{21}\right\} \\
E_{m}=\left\{v_{m 1} v_{m 2}, v_{m 2} v_{m 3}, \cdots, v_{m\left(n_{m}-1\right)} v_{m n_{m}}, v_{m n_{m}} v_{m 1}\right\} .
\end{gathered}
$$

On the basis of the above classification, we construct an odd-elegant labeling algorithm of generalized ring core network $\omega_{n_{1} n_{2} \cdots n_{m}}$ (here referred to as GRN-OEL-ALGORITHM ) and it is as follows (Table 1). 
Algorithm: GRN-OEL- ALGORITHM

Input: The number of rings $m$ of generalized ring core network $\omega_{n_{1} n_{2} \cdots n_{m}}$. The number of nodes $n_{i}(i=1,2, \cdots, m)$ of each ring $C_{n_{i}}$.

Output: Odd-elegant labeling of generalized ring core network $\omega_{n_{1} n_{2} \cdots n_{m}}$.

1. Construct a generalized ring core network $\omega_{n_{1} n_{2} \cdots n_{m}}$ with $p$ nodes and $q$ edges $\left(p=\sum_{k=1}^{m} n_{k}-m+1, q=\sum_{k=1}^{m} n_{k}\right)$ and let $n_{m+1}=0$.

2. Label the node $v_{i(2 j-1)}\left(i \in[1, m], j \in\left[1, \frac{n_{i}}{4}\right]\right)$ according to the labeling function $f\left(v_{i(2 j-1)}\right)=\sum_{k=i+1}^{m+1} n_{k}+2(j-1)$.

3. Label the node $v_{i(2 j-1)}\left(i \in[1, m], j \in\left[\frac{n_{i}}{4}+1, \frac{n_{i}}{2}\right]\right)$ according to the labeling function $f\left(v_{i(2 j-1)}\right)=\sum_{k=i+1}^{m+1} n_{k}+2 j$.

4. Label the node $v_{i(2 j)}\left(i \in[1, m], j \in\left[1, \frac{n_{i}}{2}\right]\right)$ according to the labeling function $f\left(v_{i(2 j)}\right)=\sum_{k=i+1}^{m+1} n_{k}+2 j-1$.

5. Label the edge $u v \in E$ of $\omega_{n_{1} n_{2} \cdots n_{m}}$ according to the function $g(u v)=|f(u)+f(v)| \bmod 2 q$ for $\forall u, v \in V(G)$, $u \neq v$.

6. Output a odd-elegant labeling of generalized ring core network $\omega_{n_{1} n_{2} \cdots n_{m}}$.

Then let's prove the correctness of GRN-OEL-ALGORITHM.

Theorem 1: Let $\omega_{n_{1} n_{2} \cdots n_{m}}$ be a generalized ring core network if $m, n_{1}, n_{2}, \cdots, n_{m} \in N^{*}$ and $n_{1}, n_{2}, \cdots, n_{m} \equiv 0(\bmod 4)$, then the GRN-OEL-ALGORITHM determines a odd-elegant labeling of $\omega_{n_{1} n_{2} \cdots n_{m}}$.

Proof: For every generalized ring core network $\omega_{n_{1} n_{2} \cdots n_{m}}$ if $m, n_{1}, n_{2}, \cdots, n_{m} \in N^{*}$ and $n_{1}, n_{2}, \cdots, n_{m}$ $\equiv 0(\bmod 4)$, it has $p=\sum_{k=1}^{m} n_{k}-m+1$ nodes and has $q=\sum_{k=1}^{m} n_{k}$ edges. Let $n_{m+1}=0$ and classify the nodes of $\omega_{n_{1} n_{2} \cdots n_{m}}$ into three sets $V_{1}, V_{2}, V_{3}$, then for every node of three sets label according to the labeling function $f$ proposed in the GRN-OEL-ALGORITHM :

$$
\left\{\begin{array}{l}
f\left(v_{i(2 j-1)}\right)=\sum_{k=i+1}^{m+1} n_{k}+2(j-1), \quad i \in[1, m], j \in\left[1, \frac{n_{i}}{4}\right] ; \\
f\left(v_{i(2 j-1)}\right)=\sum_{k=i+1}^{m+1} n_{k}+2 j, \quad i \in[1, m], j \in\left[\frac{n_{i}}{4}+1, \frac{n_{i}}{2}\right] ; \\
f\left(v_{i(2 j)}\right)=\sum_{k=i+1}^{m+1} n_{k}+2 j-1, \quad i \in[1, m], j \in\left[1, \frac{n_{i}}{2}\right] .
\end{array}\right.
$$

First, according to the labeling function $f$, we are able to calculate all node labeling of $\omega_{n_{1} n_{2} \cdots n_{m}}$ :

$$
\begin{gathered}
f\left(V_{1}\right)=\left\{\sum_{k=i+1}^{m+1} n_{k}+2(j-1) \mid i \in[1, m], j \in\left[1, \frac{n_{i}}{4}\right]\right\} \quad=\left\{\sum_{k=2}^{m} n_{k}, \sum_{k=2}^{m} n_{k}+2, \cdots, \sum_{k=2}^{m} n_{k}+\frac{n_{1}}{2}-2, \sum_{k=3}^{m} n_{k}, \quad \sum_{k=3}^{m} n_{k}+2, \cdots,\right. \\
\left.\sum_{k=3}^{m} n_{k}+\frac{n_{2}}{2}-2, \cdots \cdots, \quad n_{m}, n_{m}+2, \cdots, n_{m}+\frac{n_{m-1}}{2}-2,0,2,4,6, \quad \cdots, \frac{n_{m}}{2}-4, \frac{n_{m}}{2}-2\right\} . \\
f\left(V_{2}\right)=\left\{\sum_{k=i+1}^{m+1} n_{k}+2 j \mid i \in[1, m], j \in\left[\frac{n_{i}}{4}+1, \frac{n_{i}}{2}\right]\right\} \quad=\left\{\sum_{k=2}^{m} n_{k}+\frac{n_{1}}{2}+2, \sum_{k=2}^{m} n_{k}+\frac{n_{1}}{2}+4, \cdots,\right. \\
\sum_{k=2}^{m} n_{k}+n_{1}, \sum_{k=3}^{m} n_{k}+\frac{n_{2}}{2}+2, \quad \sum_{k=3}^{m} n_{k}+\frac{n_{2}}{2}+4, \cdots, \sum_{k=3}^{m} n_{k}+n_{2}-2, \sum_{k=3}^{m} n_{k}+n_{2}, \quad \cdots \cdots ; n_{m}+\frac{n_{m-1}}{2}+2, n_{m}+\frac{n_{m-1}}{2}+4, \\
\left.\cdots, n_{m}+n_{m-1}-2, n_{m}+n_{m-1}, \frac{n_{m}}{2}+2, \frac{n_{m}}{2}+4, \cdots, n_{m}-2, n_{m}\right\} . \\
f\left(V_{3}\right)=\left\{\sum_{k=i+1}^{m+1} n_{k}+2 j-1 \mid i \in[1, m], j \in\left[1, \frac{n_{i}}{2}\right]\right\}=\left\{\sum_{k=2}^{m} n_{k}+1, \sum_{k=2}^{m} n_{k}+3, \cdots, \sum_{k=2}^{m} n_{k}+n_{1}-1, \quad \sum_{k=3}^{m} n_{k}+1, \sum_{k=3}^{m} n_{k}+3, \cdots,, \cdots,\right. \\
\left.\sum_{k=3}^{m} n_{k}+n_{2}-1, n_{m}+1, n_{m}+3, \cdots, n_{m}+n_{m-1}-1,1,3, \cdots, n_{m}-1\right\} .
\end{gathered}
$$


Let the node label sets of generalized ring core network $\omega_{n_{1} n_{2} \cdots n_{m}}$ is $f(V)$, then $f(V)=\bigcup_{j=1}^{3} f\left(V_{j}\right)$. Easy to know from expressions $f\left(V_{j}\right), j \in[1,3]$ that other any two sets are disjoint sets except $f\left(v_{i 1}\right)=f\left(v_{(i+1)\left(n_{i+1}-1\right)}\right)(i \in[1, m-1])$ in number set $f\left(V_{1}\right)$ and $f\left(V_{2}\right)$. So, label function $f$ is injective from nodes set $V$ to number set $\left[0,2 \sum_{k=1}^{m} n_{k}-1\right]=[0,2 q-1]$ of generalized ring core network $\omega_{n_{1} n_{2} \cdots n_{m}}$.

Second, for $\forall e=u v \in E(G), u \neq v$ under label function $f$, let $g(e)=|f(u)+f(v)| \bmod 2 q$, then for $\forall i \in[1, m]$, we have: $g\left(v_{i 1} v_{i 2}\right)=f\left(v_{i 1}\right)+f\left(v_{i 2}\right)(\bmod 2 q)=2 \sum_{k=i+1}^{m} n_{k}+1$. Therefore, we have $g(E)=\bigcup_{i=1}^{m} g\left(E_{i}\right)=$ $\{1,3,5, \cdots, 2 q-1\}$.

So, labeling function $f$ determines a odd-elegant labeling of generalized ring core network $\omega_{n_{1} n_{2} \cdots n_{m}}$. Therefore, GRN-OEL- ALGORITHM can determine a odd-elegant labeling of $\omega_{n_{1} n_{2} \cdots n_{m}}$.

Obviously, we can get the following conclusion from theorem 1:

Theorem 1 Generalized ring core network $\omega_{n_{1} n_{2} \cdots n_{m}}$ is a odd-elegant network topology for $\forall m, n_{1}, n_{2}, \cdots, n_{m} \in N^{*}, \quad n_{1}, n_{2}, \cdots, n_{m} \equiv 0(\bmod 4)$.

\section{The implementation of odd-elegant labeling algorithm of generalized ring core network $\omega_{n_{1} n_{2} \cdots n_{m}}$}

Using Matlab language, we compile the GRN-OEL- ALGORITHM program. We finish the label experiment for sixty-eight generalized ring core network $\omega_{n_{1} n_{2} \cdots n_{m}}$ of $p$ node $(p=18,37,44,47,52,56,59$, $77,167,274,345,452,571,721,889,1068,1149,1319,1477,1595,1793,1912)$. Limited space, only the results of the label of general ring core network $\omega_{8,8,4}, \omega_{12,4,12,12}, \omega_{12,4,12,12,8}, \omega_{12,4,8,12,8,8}, \omega_{8,11,8,12,16}, \omega_{12,12,8,12,16}$, $\omega_{8,8,4,12,8,24}, \omega_{8,8,4,8,12,16,4,24}$ is given here (shown in figure 2 to figure 9). The experiment statistics CPU time of labeling and mapping for above eighteen generalized ring core network $\omega_{n_{1} n_{2} \cdots n_{m}}$ and analyze development trend of CPU time of labeling and mapping for generalized ring core network $\omega_{n_{1} n_{2} \cdots n_{m}}$. Statistic and analysis results of experimental are shown in table 2 and figure 10, figure 11.

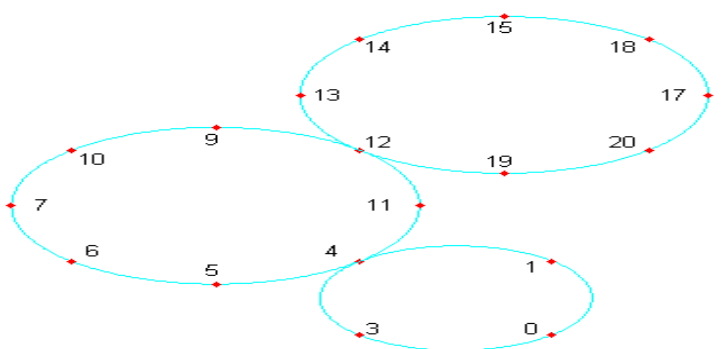

Fig. 2. Odd-elegant labeling of $\omega_{8,8,4}$

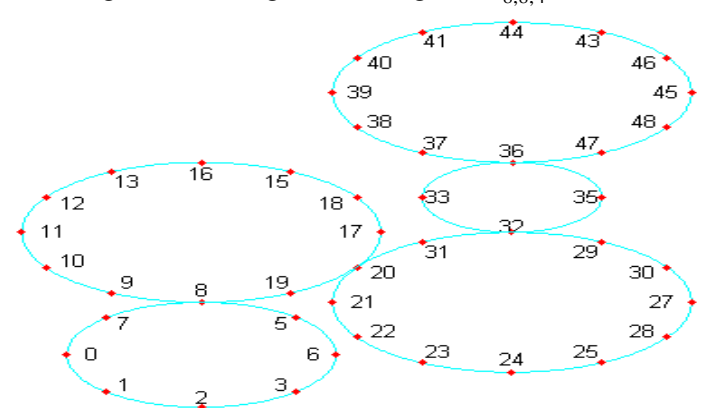

Fig. 4. Odd-elegant labeling of $\omega_{12,4,12,12,8}$

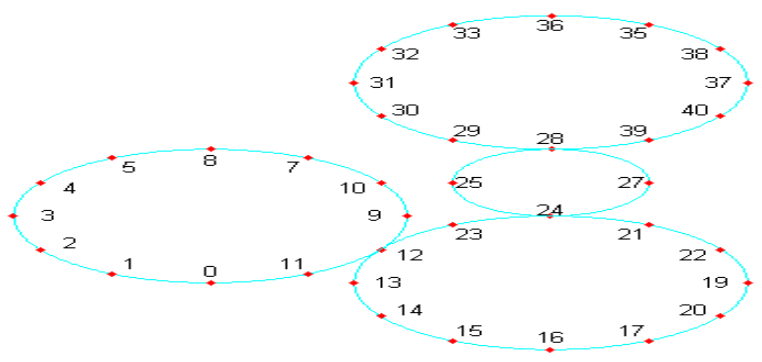

Fig. 3. Odd-elegant labeling of $\omega_{12,4,12,12}$

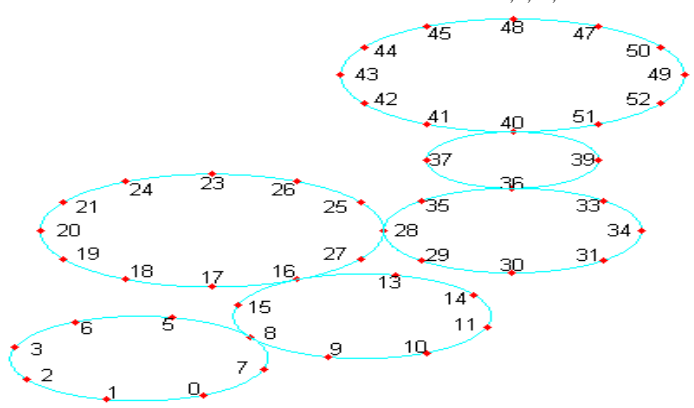

Fig. 5. Odd-elegant labeling of $\omega_{12,4,8,12,8,8}$ 


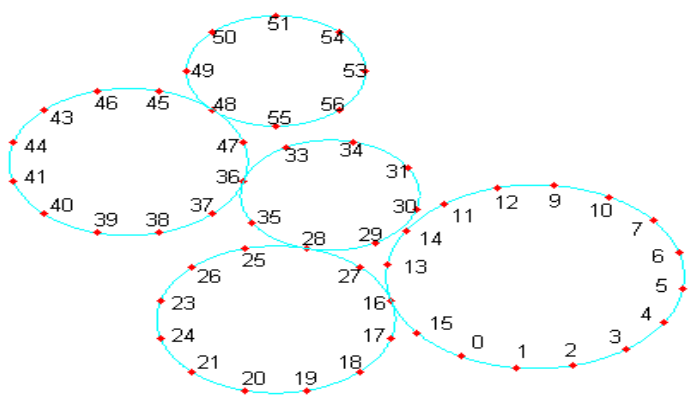

Fig. 6. Odd-elegant labeling of $\omega_{8,12,8,12,16}$

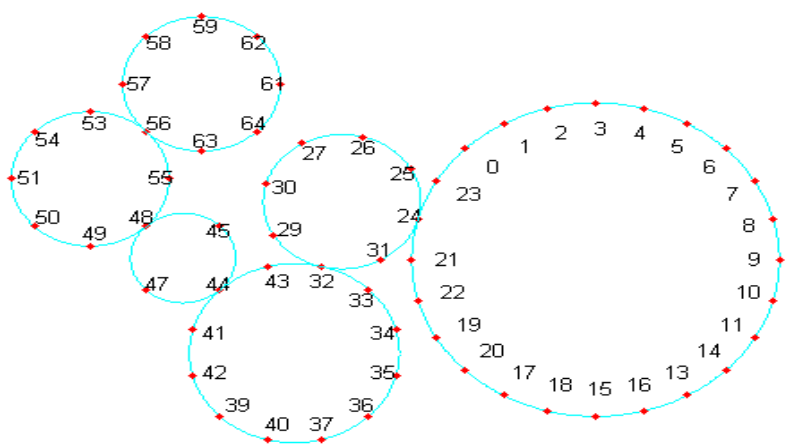

Fig. 8. Odd-elegant labeling of $\omega_{8,8,4,12,8,24}$

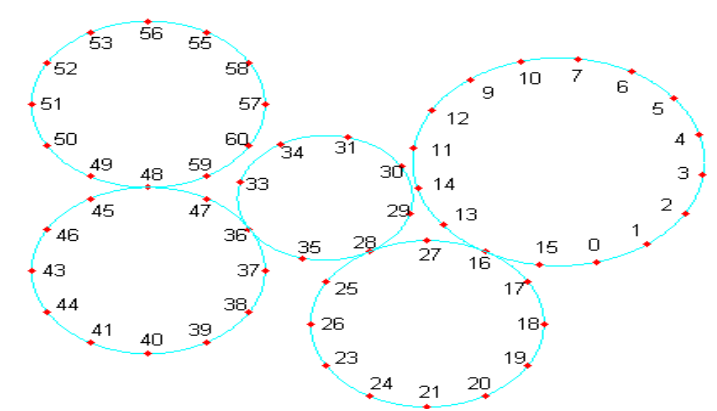

Fig. 7. Odd-elegant labeling of $\omega_{12,12,8,12,16}$

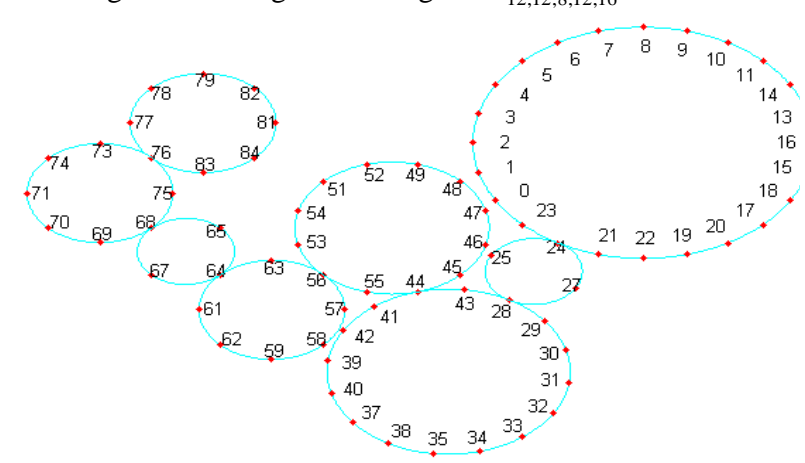

Fig. 9. Odd-elegant labeling of $\omega_{8,8,4,8,12,16,4,24}$

TABLE 2. CPU TIME STATISTICS OF ODD-ELEGANT LABELING AND MAPPING LABELING OF GENERALIZED RING CORE NETWORK $G=\omega_{n_{1} n_{2} \cdots n_{m}}$

\begin{tabular}{|c|l|c|c|}
\hline vertex number of $\boldsymbol{G}$ & \multicolumn{1}{|c|}{ generalized ringcore network $\boldsymbol{G}$} & $\begin{array}{c}\text { CPU time of } \\
\text { labeling of } \boldsymbol{G}\end{array}$ & $\begin{array}{c}\text { CPU time of } \\
\text { mapping } \boldsymbol{G}\end{array}$ \\
\hline 18 & $\omega_{8,8,4}$ & 0.000043 & 0.182989 \\
\hline 37 & $\omega_{12,4,12,12}$ & 0.000053 & 0.204855 \\
\hline 44 & $\omega_{12,4,12,12,8}$ & 0.000057 & 0.195147 \\
\hline 47 & $\omega_{12,48,12,8,8}$ & 0.000057 & 0.201063 \\
\hline 52 & $\omega_{8,12,8,12,16}$ & 0.000060 & 0.196301 \\
\hline 56 & $\omega_{12,12,8,12,16}$ & 0.000059 & 0.201579 \\
\hline 59 & $\omega_{8,8,4,12,8,24}$ & 0.000063 & 0.202551 \\
\hline 77 & $\omega_{8,8,4,8,12,16,4,24}$ & 0.000072 & 0.217822 \\
\hline 167 & $\omega_{8,8,4,8,12,16,16,24,40,40}$ & 0.000095 & 0.257083 \\
\hline 274 & $\omega_{8,8,4,8,12,16,4,24,40,80,80}$ & 0.000188 & 0.278325 \\
\hline 345 & $\omega_{8,8,4,8,12,16,16,24,60,120,40,40}$ & 0.000153 & 0.304310 \\
\hline 452 & $\omega_{8,8,4,8,12,16,4,24,40,80,80,10,80}$ & 0.000184 & 0.338296 \\
\hline 571 & $\omega_{8,8,4,8,12,16,4,24,40,80,80,100,80,120}$ & 0.000278 & 0.381158 \\
\hline 721 & $\omega_{8,8,4,8,12,16,16,24,60,120,60,80,60,80,100,80}$ & 0.000265 & 0.449175 \\
\hline 889 & $\omega_{8,8,4,8,12,16,4,44,40,80,80,100,80,120,120,200}$ & 0.000383 & 0.483294 \\
\hline 1068 & $\omega_{8,8,4,4,12,16,4,24,40,80,80,100,80,120,120,200,180}$ & 0.000390 & 0.548901 \\
\hline 1149 & $\omega_{8,8,4,8,12,16,4,24,40,80,80,10,80,100,100,140,100,140,120,100}$ & 0.000421 & 0.670272 \\
\hline 1319 & $\omega_{8,8,4,8,12,16,4,24,40,80,80,100,80,100,100,140,100,140,120,100,132,40}$ & 0.000495 & 0.776347 \\
\hline 1477 & $\omega_{8,8,4,8,12,16,4,24,40,80,80,100,80,100,100,140,100,140,120,100,132,40,120,40}$ & 0.000557 & 0.904812 \\
\hline 1595 & $\omega_{8,8,8,4,12,16,4,24,40,80,80,100,80,100,100,140,100,140,120,100,132,40,120,40,40,80}$ & 0.000615 & 1.03266 \\
\hline 1793 & $\omega_{8,8,4,8,12,16,4,24,40,80,80,100,80,100,100,140,100,140,120,100,132,40,120,40,40,80,80,120}$ & 0.000695 & 1.19125 \\
\hline 1912 & $\omega_{8,8,4,8,12,16,4,24,40,80,80,10,80,100,100,140,100,140,120,100,132,40,120,40,40,80,80,120,120}$ & 0.000757 & 1.27448 \\
\hline
\end{tabular}




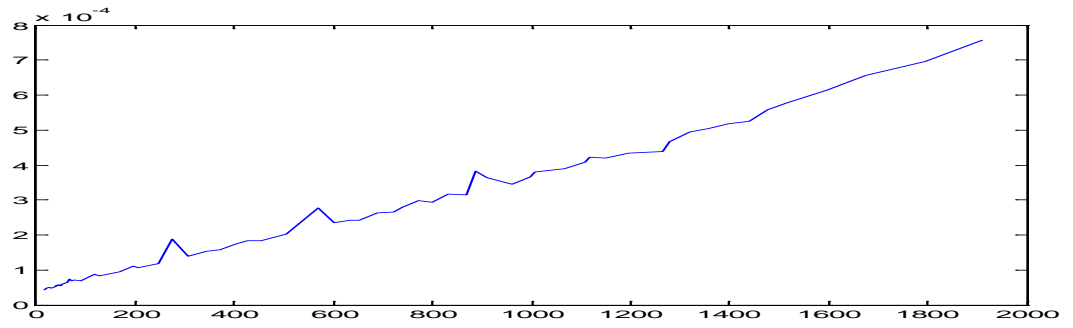

Fig. 10. The CPU time trend chart of odd-elegant labeling of generalized ring core network $\omega_{n_{1} n_{2} \cdots n_{m}}$

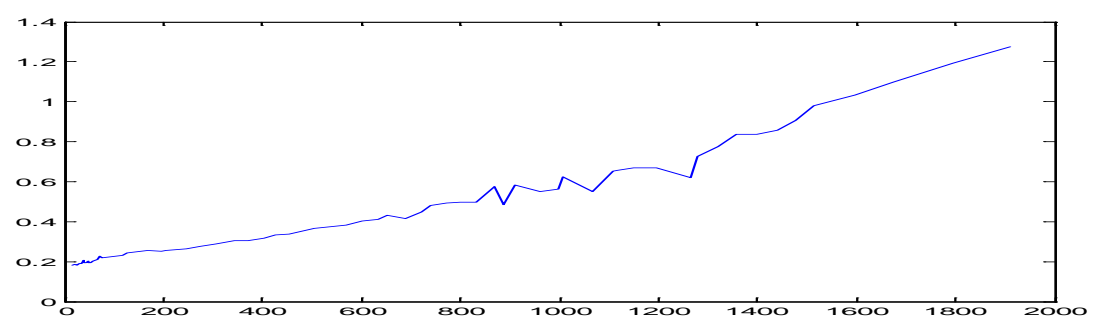

Fig. 11. The CPU time trend chart of mapping odd- elegant labeling graph of generalized ring core network $\omega_{n_{1} n_{2} \cdots n_{m}}$

According to the change trend of figure 10, we find that function relation between CPU time of labeling for generalized ring core network $\omega_{n_{1} n_{2} \cdots n_{m}}$ and vertex number $p$ of generalized ring core network $\omega_{n_{1} n_{2} \cdots n_{m}}$ is in an approximate linear relationship. We also find that function relation between CPU time of mapping odd-elegant labeling graph of generalized ring core network $\omega_{n_{1} n_{2} \cdots n_{m}}$ and vertex number $p$ of generalized ring core network $\omega_{n_{1} n_{2} \cdots n_{m}}$ is in an approximate linear relationship by figure 11. Therefore, we may safely draw the conclusion that the GRN-OEL-ALGORITHM is effectiveness.

\section{Conclusion}

The computer implementation of odd-elegant labeling algorithm of special network topology structure has practical guiding significance to computer communication network system design of functional, reliability, low communication cost. In this paper, the author defines generalized ring core network $\omega_{n_{1} n_{2} \cdots n_{m}}$, designs the GRN-OEL- ALGORITHM when $n_{1}, n_{2}, \cdots, n_{m} \equiv 0(\bmod 4)$, proves odd-elegant of this network topology, works out the corresponding software.

\section{Acknowledgment}

This research was supported completely by the National Natural Science Foundation of China under Grant No. 61163054, No. 61363060 and No. 61163037; Research Projects of Gansu Province Education Science "Twelfth Five" project No. GS[2015]GHB0174 and No. GS[2013]GHB0930.

\section{References}

[1] G. J. Chang, D. F. Hsu, and D. G. Rogers, “Additive variations on a graceful theme: some results on harmonious and other related graphs”, Congr. Numer., 1981, pp.181-197.

[2] X.-Q. Zhou, B.Yao, and X.-E. Chen, "Every lobster is odd- elegant ”, Information Processing Letters, 113 (2013) 30-33.

[3] M.Z.Youssef, “On E k-cordial labeling”, Ars Combin., 2014, pp.271-279.

[4] X.-Q. Zhou, B.Yao, X.-E. Chen, and H.-X. Tao, “A proof to the odd-gracefulness of all lobsters ”, Ars Combin., 103 (2012) 13-18. 
[5] W.Feng, and C.Xu, “A survey of the gracefulness of digraphs ”, Int. J. Pure Appl. Math., 69 (2011) 245-253.

[6] M.Hussain, E.T. Baskoro, and K.Ali, “On super antimagic total labeling of Harary graph ”, Ars Combin., 104 (2012) 225-233.

[7] Z.Gao, “Odd graceful labelings of some union graphs ”, Nat. Sci. Heilongjiang Univ., 24 (2007) 35-39.

[8] C. Barrientos, “Odd-graceful labelings of trees of diameter 5 ”, Graphs Comb., 2009, pp. 307-313. 